\title{
The impact of clouds on image parameters in IACT at very high energies
}

\author{
Dorota Sobczyńska a and Włodek Bednarek \\ University of Łódź, Department of Astrophysics, Pomorska 149/153, 90-236 Łódź, Poland
}

\begin{abstract}
The effective observation time with the Cherenkov telescopes arrays is limited to clear sky conditions due to considerable absorption of Cherenkov light by the possible presence of clouds. However below the cloud altitude the primary particles with high energies can still produce enough Cherenkov photons to allow the detection by the large telescopes. In this paper, using the standard CORSIKA code, we investigate the changes of image parameters due to the absorption of Cherenkov radiation by the cloud (for $\gamma$-ray and proton showers with various energies - from $2 \mathrm{TeV}$ to $100 \mathrm{TeV}$ and from $10 \mathrm{TeV}$ to $200 \mathrm{TeV}$, respectively). We consider the clouds with different transmissions located at various altitudes above the ground level (between $8 \mathrm{~km}$ and $3 \mathrm{~km}$ ). We show that, for both simulated primary particles at fixed energy, the WIDTH and the DIST distributions are shifted towards larger values in the presence of clouds in comparison to the clear sky simulations. This shift decreases with the cloud altitude. The LENGTH distributions are shifted towards smaller values for images of primary $\gamma$-rays, while for primary protons this shift is not observed. We conclude that the large Cherenkov telescopes with large camera FOV could be used for observation of $\gamma$-ray showers with high energies in the presence of clouds.
\end{abstract}

\section{Introduction}

The imaging air Cherenkov technique (IACT) has been successfully used to record $\gamma$-rays from cosmic sources. The two dimensional angular distribution of the Cherenkov light produced by the shower (so called shower image) is measured using IACT. Parameters which describe shower images were proposed by Hillas in 1985 [1]. He also showed a method of $\gamma /$ hadron separation based on those parameters.

The observations using the Cherenkov telescopes are carried out only during nights because the amount of Cherenkov light created by the shower is relatively small. The presence of clouds disturbs observations due to the fact that Cherenkov photons are absorbed and scattered by molecules of clouds. As a result, the amount of light can be too small to trigger a telescope. Additionally, the recorded images can be distorted. This may lead to deterioration in the sensitivity of the telescope or telescope arrays. Therefore the effective time of observation is limited to clear sky conditions. Currently operating experiments like HESS [2], MAGIC [3] and VERITAS [4] monitor the atmosphere during data taking.

The influence of the atmospheric parameters on the IAC technique has been already studied and presented in [5]. The reconstructed energy of the shower and estimated fluxes of primary particles (from the HESS data) can be corrected for the presence of low-level aerosols [6]. The data taken by MAGIC during nights with additional partial absorption (caused by aerosols at a relatively low level) are also corrected $[7,8]$. However the applied method is based on the correction of the image parameter called SIZE. It has been shown in $[9,10]$, that also other Hillas parameters can be affected by fully opaque clouds.

In this paper we investigate the influence of cloud parameters (its altitude and transmission) on the Hillas

\footnotetext{
a e-mail: dsobczynska@uni.lodz.pl
}

parameters, which describe the shape and the position of the image. We studied the problem for the $\gamma$-ray and proton initiated showers with different fixed energies. Based on Monte Carlo (MC) simulations, we show DIST, WIDTH and LENGTH distributions, which are expected in the presence of various clouds, for a detector area of $225 \mathrm{~m}^{2}$. We show that the changes of the image parameters are similar for primary $\gamma$-rays and protons. Therefore the $\gamma$ ray selection, based on the presented image parameters, should be possible for the data collected under cloud cover. We conclude that large mirror Cherenkov telescopes with large field of view (FOV) camera should allow for detection of very high energy $\gamma$-ray showers. Large mirrors are needed because of significant Cherenkov light absorption by molecules of clouds. Cameras with large FOV are required because the images may be significantly shifted outwards the camera center.

\section{Monte Carlo simulations}

We have used the CORSIKA code version 6.99 [12]) in order to simulate the shower development in the atmosphere. Cherenkov photons (created by charged particles from the shower) were absorbed in processes of the Rayleigh and Mie scattering in the atmosphere according to the Sokolsky formula [13]. Simulations have been performed for vertical showers at the MAGIC site [3] (the altitude of $2.2 \mathrm{~km}$ a.s.1.). To determine the two dimensional angular distribution of the Cherenkov light, we simulated a telescope with a mirror area of $225 \mathrm{~m}^{2}$ for eight different impact parameters (between $22.5 \mathrm{~m}$ and $322.5 \mathrm{~m}$ ). The histograms of angular distributions were obtained by counting the Cherenkov photons in the ranges $-1^{\circ}$ and $14^{\circ}$ (in the $\mathrm{x}$ axis) and $-7.5^{\circ}$ to $7.5^{\circ}$ (in the $y$ axis). The typical pixel size in operating IACT is approximately $0.1^{\circ}$. This value was used as the width bin of the histogram.

This is an Open Access article distributed under the terms of the Creative Commons Attribution License 4.0, which permits unrestricted use, distribution, and reproduction in any medium, provided the original work is properly cited. 

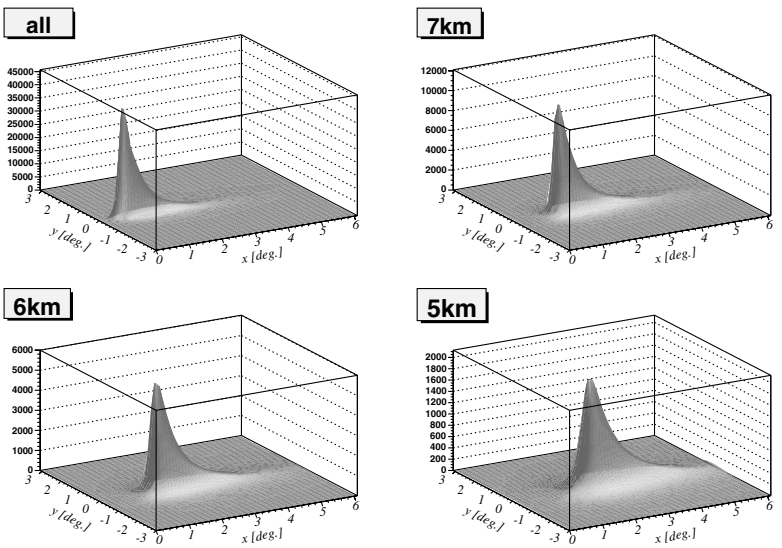

Figure 1. The expected mean images of showers initiated by $20 \mathrm{TeV} \gamma$-rays with the impact parameter $112.5 \mathrm{~m}$ for different altitudes of clouds. Note the different values on the z-axes.

The simulations have been performed without the presence of any clouds and with clouds at the different altitudes equal to $10,7,6$ and $5 \mathrm{~km}$ a.s.l.. Those values have been chosen because they correspond to the heights, which are above and below the shower maximum in the investigated energy range [9]. For each cloud altitude we applied different transmission of the cloud (T), starting from $\mathrm{T}=0$ (fully opaque) up to $\mathrm{T}=1$ (transparent) with a step size of 0.2 . Cherenkov photons, produced in the shower above the cloud level, were partially absorbed (with the probability equal to the cloud transmission). We have simulated $\gamma$-ray showers with energies of 2,5 , $10,20,50 \mathrm{TeV}$ and $100 \mathrm{TeV}$. The proton initiated showers have been simulated with energies of 10, 20, 50, 100 and $200 \mathrm{TeV}$. The number of simulated events for each cloud parameter was 1000 apart from $\gamma$-rays at $100 \mathrm{TeV}$ and protons at $200 \mathrm{TeV}$ (500 events).

\section{Results and discussion}

The average images of the $20 \mathrm{TeV}$ primary $\gamma$-rays at impact parameter $112.5 \mathrm{~m}$ are presented in Fig. 1 for the case of clear sky (marked by "all" in Fig. 1) and fully opaque clouds at 7,6 and $5 \mathrm{~km}$ a.s.1.. In the presence of clouds the images contain less Cherenkov light and they are less concentrated in comparison to clear sky simulations. Images are also shifted away from the camera center (located at the position of $\left.\left(0^{\circ}, 0^{\circ}\right)\right)$ due to the fact that those Cherenkov photons which have been absorbed by the clouds are produced higher in the atmosphere. There are two important effects in the higher layers of the atmosphere. First, the Cherenkov angle is smaller. Second, the angular distribution of charged particles is narrower. As a result, we expect that Cherenkov photons with very small angles are not recorded on the camera for fully opaque clouds. Therefore a part of the image close to the camera center is missing (so called the geometrical effect). Finally, showers can not be detected by cameras with small FOV. The Hillas parameters should change in the presence of clouds in comparison to clear sky conditions.

The impact of a cloud cover on the shower images depends on the impact parameter of the shower because the density of Cherenkov light is correlated with the height of production of Cherenkov photons in the $\gamma$-ray initiated shower [11]. The Hillas parameters called DIST, WIDTH and LENGTH describe the position and the shape of the image. They were calculated in few steps after the MC simulations. First, we added the night sky background (NSB) (with the level measured on La Palma [14]) to the angular distributions of the Cherenkov light obtained from MC simulations. Next, the images were cleaned. In the calculations we neglected all pixels with a signal below 30 photons. Afterwards, image parameters were calculated for all eight simulated impact parameters. Finally, the distribution of the Hillas parameters was interpolated in the range of impact parameters between $32.5 \mathrm{~m}$ and $322.5 \mathrm{~m}$ for each simulation. All distributions presented below were obtained for images with a total signal (SIZE) above 200 photons.

In Fig. 2 we show the dependencies of the Hillas parameters on the transparency of the cloud located at a height equal to $6 \mathrm{~km}$ a.s.1.. The DIST, WIDTH and LENGTH distributions are presented as the top, middle and bottom panel of figures, respectively. Four columns of figures demonstrate different primary particle energy from the left to right: $5 \mathrm{TeV} \gamma$-rays, $50 \mathrm{TeV} \gamma$-rays, $10 \mathrm{TeV}$ proton and $100 \mathrm{TeV}$ proton. Protons and $\gamma$-rays with low and high energies are shown in order to indicate possible differences in the impact of cloudiness on the expected image parameters.

The distributions of the DIST parameter of the $\gamma$-ray and proton initiated showers become wider. They are more shifted towards larger angles for lower cloud transmissions (see Figs. 2a-d). At low energy (both primary particles), the shift of the DIST distributions is significantly larger for the fully opaque clouds due to the geometrical effect.

The distributions of the WIDTH parameter become wider with decreasing transmission of the cloud for all simulated primary particles and energies (see Figs. 2e-h). The shift of the WIDTH distribution of the $\gamma$-ray and proton showers towards larger values is expected for higher energies. For lower energies a similar shift is obtained only for the cloud transparency of 0 (which is due to the same geometrical effect).

The distributions of the LENGTH parameter of the $\gamma$ ray and proton images are shifted towards larger angles for lower cloud transmission, except fully opaque clouds (see Figs. 2i-1)). In this case, the LENGTH distributions of primary $\gamma$-rays are significantly shifted towards lower angles in comparison to the clear sky simulations due to geometrical effects. This shift is not expected for the primary protons (Figs. 2k,1). Similar changes of the distributions of Hillas parameters with the cloud transmission were obtained also for the cloud altitude of $7 \mathrm{~km}$ and $5 \mathrm{~km}$. In the case of the cloud located at $10 \mathrm{~km}$ a.s.l., the expected distributions of the image parameters are very similar to the clear sky simulations for all investigated transparencies.

We present dependencies of the Hillas parameters on the cloud altitude for the fully opaque cloud (see Fig. 3). The cloud transmission of 0 was chosen for demonstration purposes because it shows an extreme case of the sky conditions. Black and solid lines in all figures are the results of the clear sky simulations. Panels and columns of this Figure correspond to those in Fig. 2. The distributions 

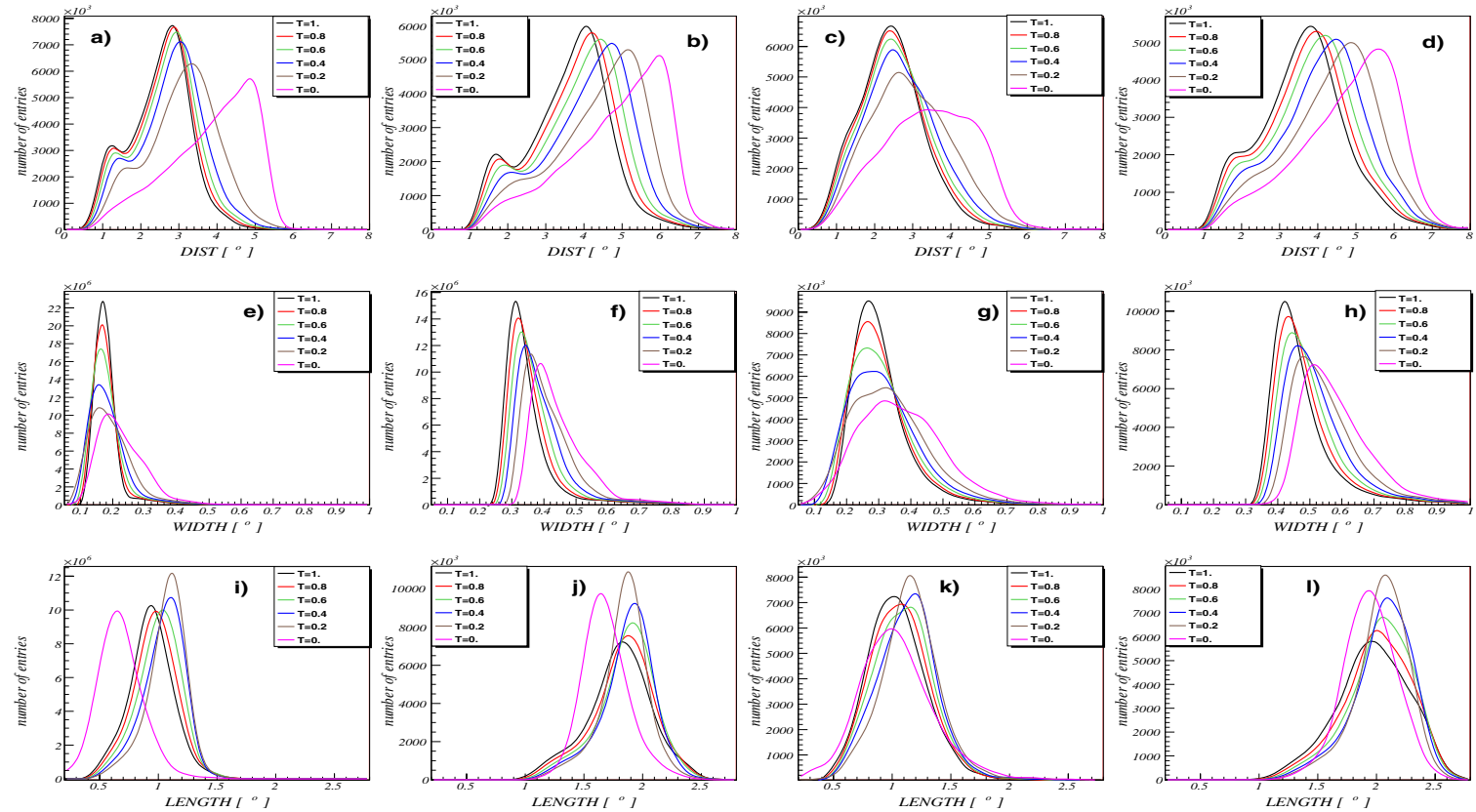

Figure 2. Distributions of the Hillas parameters for the cloud at $6 \mathrm{~km}$ a.s.1.. Different colors of smooth histograms correspond to various simulated transparencies (from 1 to 0 ). Primary $\gamma$-rays with energies of $5 \mathrm{TeV}$ and $50 \mathrm{TeV}$ are shown in the first and second column, respectively. Primary proton with energies $10 \mathrm{TeV}$ and $100 \mathrm{TeV}$ are presented in the third and last column, respectively.
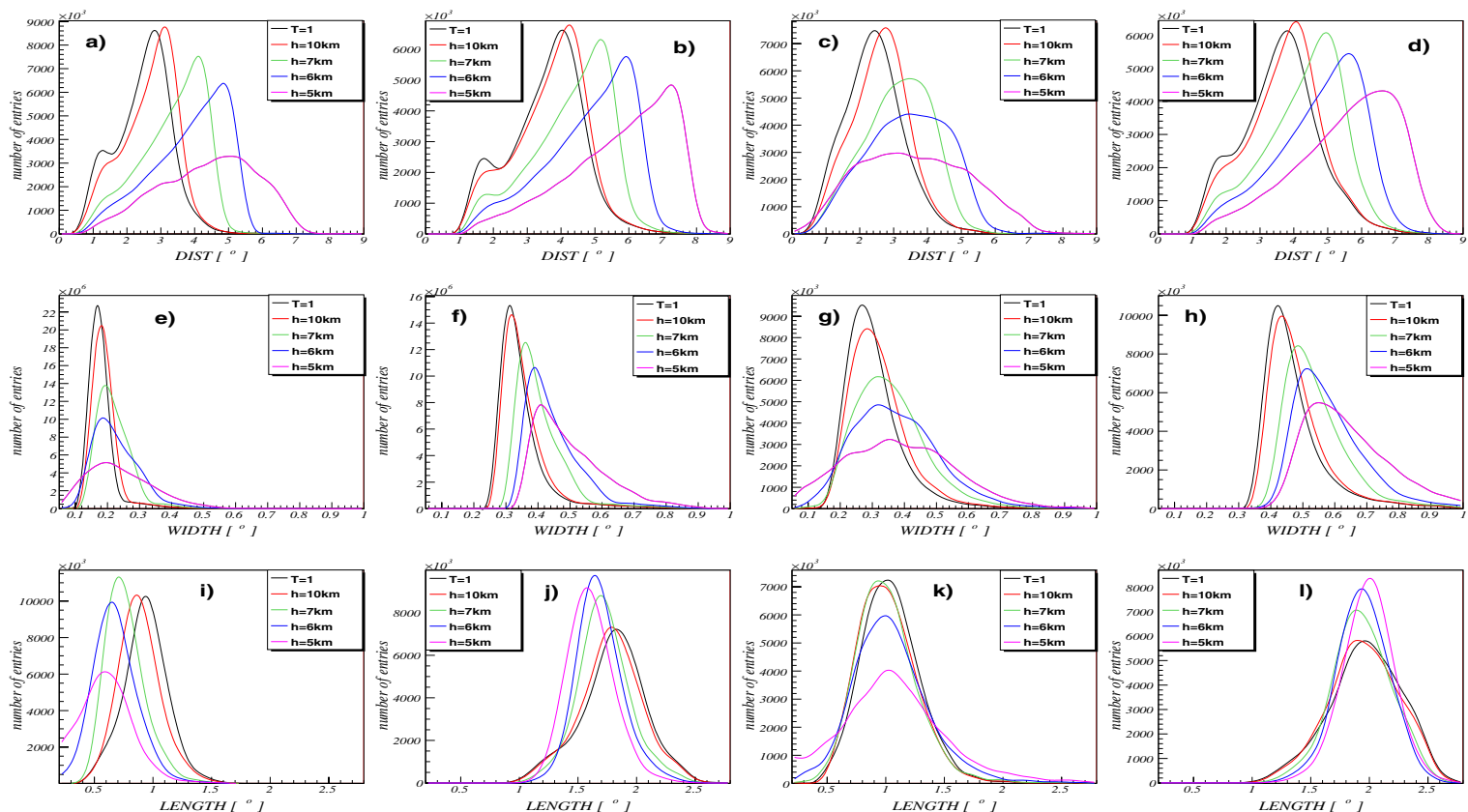

Figure 3. Distributions of the Hillas parameters for the clear sky and fully opaque clouds. Different colors of smooth histograms correspond to various simulated cloud altitudes. Primary $\gamma$-rays with energies of $5 \mathrm{TeV}$ and $50 \mathrm{TeV}$ are shown in the first and second column, respectively. Primary proton with energies $10 \mathrm{TeV}$ and $100 \mathrm{TeV}$ are presented in the third and last column, respectively.

of the DIST parameter of the $\gamma$-ray and proton induced showers (Figs. 3a-d) become wider. They are shifted towards larger angles for lower cloud altitude, except for protons with low energy (Fig. 3c). In this case, shifts of the DIST distribution are similar for cloud altitudes of 5 and $6 \mathrm{~km}$ a.s.1.. The WIDTH distributions also become wider with decreasing altitude of the cloud (see Figs. 3e-h). The expected shift (towards larges angles) is smaller at lower energies than at higher energies for both simulated primary particles. As expected, the changes of DIST and WIDTH distributions are larger for lower altitudes of fully opaque clouds. There are differences between the influences of clouds (with $\mathrm{T}=0$ ) on the LENGTH distributions of the $\gamma$-ray and proton initiated showers (see Figs. 3i-1). The distributions of the LENGTH parameter of the $\gamma$-rays are significantly shifted towards smaller angles for lower altitudes of clouds (Figs. 3i,j). This shift was not obtained for primary protons at low energy (Fig. $3 \mathrm{k}$ ), while in the 
high energy range a small shift towards larger angles with decreasing cloud altitude is observed (Fig. 31).

We have checked the influence of the altitude of clouds (with transmissions larger than 0) on the distributions of the Hillas parameters. The changes of the Hillas parameters due to cloudiness are similar for images of the primary $\gamma$-rays and proton initiated showers. The distributions of DIST and LENGTH are shifted towards larger values for lower cloud altitude. The changes of the WIDTH parameter depend on the simulated energy region. The WIDTH distributions are shifted in the same direction for high energies in contrast to low energies (shifts to the smaller angles were obtained) for lower cloud altitude. We have checked that, the differences between low and high energies are correlated with the position of clouds with respect to the shower maximum.

\section{Conclusions}

The presence of clouds influences the expected image of the shower because Cherenkov light is absorbed by the particles forming the clouds. As the result, images contain less light. They are shifted in the camera plane towards larger angles. Therefore, IACT equipped with large mirror (to keep reasonable high trigger rate) and with the cameras with large FOV (to record shifted image) are needed to observe $\gamma$-rays sources when the sky is covered by clouds.

As expected, the larger changes of the expected DIST, WIDTH and LENGTH distributions were obtained for the lower altitude and lower transmission of the cloud. The presence of clouds (above $10 \mathrm{~km}$ a.s.1.) almost do not influence the image parameters of the primary $\gamma$-ray and proton initiated showers at high energies. This altitude of clouds is above the shower maximum for all simulated primary energies and particles. For clouds at lower altitudes, the changes of the image parameters can be explained by two effects: decreasing the image concentrations (due to additional absorption) and the geometrical effect. The second effect strongly depends on the differences between altitudes of the shower maximum and the cloud. The stronger impact of the cloudiness on the image parameters is obtained for altitudes of the clouds close and below the shower maximum.
The $\gamma$ /hadron separation is based on the differences between the distributions of image parameters, but the method is much more sensitive on the WIDTH than on the LENGTH. The impact of the cloudiness on the WIDTH distributions is similar for $\gamma$-rays and proton induced showers in all simulated cases. Therefore, we expect, that $\gamma$-ray events can be selected from the hadronic background at very high energies even in the presence of clouds.

We have shown in [10], that for camera with FOV limited to $8^{\circ}$ the $\gamma$ /hadron separation is still possible. The Middle Size Telescope (MST) equipped with such cameras will be built in the project CTA [15]. They might be used for observations during cloud presence.

The influence of the cloud presence on an expected trigger rate, a collection area, an angular resolution, an energy resolution and a sensitivity curve will be considered in the future studies for a particular telescope or IACT system.

This work was supported by Polish Grant from Narodowe Centrum Nauki 2011/01/M/ST9/01891.

\section{References}

[1] Hillas A.M., Proc. 19th ICRC vol 3, 445 (1985)

[2] Aharonian F. et al, A\&A 457, 899 (2004)

[3] Aleksić J. et al, Astropart. Phys. 35, 435 (2012)

[4] Weekes T.C. et al, Astropart. Phys. 17, 221 (2002)

[5] Bernlöhr K., Astropart. Phys. 12, 255 (2000)

[6] Nolan S.J. et al, Astropart. Phys. 34, 304 (2010)

[7] Dorner D. et al, A\&A 493, 721 (2009)

[8] Fruck C. et al, 33th ICRC, [arXiv:1403.3591] (2013)

[9] Sobczynska D. and Bednarek W., 33th ICRC ID0335 (2013)

[10] Sobczynska D. and Bednarek W., accepted in J. Phys. G (2014)

[11] Sobczynska D., J. Phys. G 36, 045201 (2009)

[12] Heck D. et al, Technical Report FZKA 6019 (Forschungszentrum Karlsruhe, 1998)

[13] Sokolsky P., Introduction to Ultrahigh Energy Cosmic Ray Physics (Addison-Wesley, 1989)

[14] Mirzoyan R. and Lorenz E., MPI-PhE/94-35 (preprint, Munich, 1994)

[15] Actis et al, Exper. Astron. 32, 193 (2011) 